\title{
6. Unbezahlte Sorgetätigkeiten von Frauen und Männern im Verlauf der zweiten Lebenshälfte
}

\author{
Daniela Klaus und Claudia Vogel
}

\section{Kernaussagen}

Die Wahrscheinlichkeit unbezahlter Sorgetätigkeiten steigt im Verlauf der zweiten Lebenshälfte zunächst an, sinkt danach bis zum 90. Lebensjahr wieder ab: Dabei zeigt sich für die Enkelbetreuung eine Konzentration auf die späte Erwerbsphase sowie auf das gesetzliche Renteneintrittsalter. Pflege- und Unterstützungsleistungen kommen ab dem 50. Lebensjahr bis zum Ende der Erwerbsphase besonders häufig vor, verteilen sich aber insgesamt gleichmäßiger über die zweite Lebenshälfte als die Enkelbetreuung.

Frauen übernehmen unbezahlte Sorgetätigkeiten mit höherer Wahrscheinlichkeit als Männer: Im Alter von 60 Jahren ist die Wahrscheinlichkeit der Enkelbetreuung für Frauen um zehn Prozent höher als für Männer. Bei Pflege- und Unterstützungsleistungen liegt der Unterschied zwischen Frauen und Männern bei sieben Prozent.

Frauen übernehmen unbezahlte Sorgetätigkeiten biografisch früher als Männer: Bei Frauen ist die Wahrscheinlichkeit für die Betreuung von Enkelkindern im Alter von 63 Jahren am höchsten, für Männer im Alter von 67 Jahren. Auch Pflege- und Unterstützungsleistungen werden von den Frauen früher erbracht als von den Männern, nämlich verstärkt im Alter von 57 Jahren gegenüber 61 Jahren bei den Männern.

Unbezahlte Sorgetätigkeiten von Frauen und Männern entwickeln sich im Kohortenvergleich unterschiedlich: Frauen und Männer späterer Geburtskohorten betreuen Enkelkinder in zunehmend höherem Alter. Pflege- und Unterstützungsleistungen werden bereits eher in der zweiten Lebenshälfte übernommen als bei früher geborenen Kohorten und zudem zunehmend häufiger. Geschlechterunterschiede bei der Enkelbetreuung nehmen über die Geburtskohorten hinweg ab, während Frauen weiterhin mit höherer Wahrscheinlichkeit und biografisch früher Pflege- und Unterstützungsleistungen übernehmen als Männer. 


\subsection{Einleitung}

Unbezahlte Arbeiten wie Hausarbeit und Sorgetätigkeiten für (Familien-)Angehörige, Nachbarinnen und Nachbarn sowie für Personen aus dem Freundes- oder Bekanntenkreis stoßen auf große Nachfrage. Sie werden von Privatpersonen in Privathaushalten erbracht und sie sind von hohem Wert: für das individuelle Wohlbefinden der Empfängerinnen und Empfänger, für die soziale Einbindung der beteiligten Personen, die Pflege ihrer Solidargemeinschaft sowie für die gesamtgesellschaftliche Wohlfahrtsproduktion. Eine Schätzung zur Wertschöpfung durch unbezahlte Arbeit weist aus, dass sie fast 40 Prozent der im Bruttoinlandsprodukt enthaltenen Bruttowertschöpfung ausmacht (2012/2013), wobei ihr Wert auf jährlich 987 Milliarden Euro geschätzt wird (Schwarz \& Schwahn 2016: 46). Der Großteil davon geht auf Arbeiten im Haus(-halt) und Garten zurück (79 Prozent) und weitere neun Prozent resultieren aus Pflege- und Betreuungsaufgaben (ebd.: 47).

Dabei wird unbezahlte Arbeit keineswegs nur in früheren Lebens- und Familienphasen erbracht. Auch Menschen mittleren und hohen Alters übernehmen solche Aufgaben in beträchtlichem Umfang. Neben (gegenseitigen) Hilfen bei Reparaturen, Garten- und Hausarbeit beinhaltet das auch die Unterstützung bedürftiger Personen. Das kann in Form finanzieller Zuwendungen für Familienmitglieder sein, als Betreuung und Versorgung von Enkelkindern oder als Fürsorge für Angehörige, die aufgrund gesundheitlicher Beschwerden in ihrer Alltagsorganisation eingeschränkt oder pflegebedürftig sind. So haben Auswertungen des Deutschen Alterssurveys (DEAS) gezeigt, dass die Übernahme von Enkelbetreuung nach einem rückläufigen Trend der letzten Jahre (von 34 Prozent im Jahr 1996 auf 25 Prozent im Jahr 2008) wieder zugewonnen hat: Im Jahr 2014 berichten etwa 30 Prozent aller Großeltern, dass sie Enkelkinder betreuen (Mahne \& Klaus 2017: 241). Auch ist der Anteil derer, die gesundheitlich beeinträchtigte Personen unterstützen oder pflegen, im Laufe der letzten Jahre gestiegen: von zwölf Prozent
(1996) auf 16 Prozent (2014) (Klaus \& Tesch-Römer 2017: 191).

Diese (erneut) wachsenden Betreuungs- und Unterstützungsquoten im privaten Bereich sind Antwort auf eine gestiegene Nachfrage. Diese hat bislang keine ausreichende Entlastung durch die Schaffung (ergänzender) professioneller und institutioneller Betreuungs- und Versorgungsmöglichkeiten erfahren. Hier findet die in ihren Grundzügen konservative Wohlfahrtspolitik Deutschlands ihren Ausdruck, die in den vergangenen Jahrzehnten nur punktuell modifiziert wurde. Insbesondere Sozial- und Pflegepolitik fußen weiterhin auf dem Subsidiaritätsprinzip, wonach bei Hilfebedürftigkeit die Erstverantwortung in der Familie liegt. So werden 1,4 der aktuell 2,9 Millionen Menschen, die im Sinne der Pflegeversicherung leistungsberechtigt sind, ausschließlich durch Angehörige im häuslichen Bereich versorgt (Statistisches Bundesamt 2017: 5). Darüber hinaus wird geschätzt, dass weitere 5,4 Millionen Menschen zwar nicht pflegebedürftig gemäß dieser Definition sind, dennoch auf Grund gesundheitlicher Einschränkungen auf regelmäßige Unterstützung angewiesen sind (Geyer \& Schulz 2014). Mehr noch für sie als für die im engeren Sinne Pflegebedürftigen ist davon auszugehen, dass deren Unterstützung überwiegend im privat-informellen $\mathrm{Be}$ reich organisiert und erbracht wird.

Zumindest einige Entlastungen brachten neben der Ausweitung der Pflegeversicherung vor allem familien- und arbeitsmarktpolitische Maßnahmen, welche die Förderung der Erwerbstätigkeit von Müttern zum Ziel haben, bei gleichzeitiger Verbesserung der Vereinbarkeit mit Sorgetätigkeiten (vgl. auch den Zweiten Bericht zur Gleichstellung von Frauen und Männern: Deutscher Bundestag 2017). So wurde der Ausbau von Kinderbetreuungseinrichtungen weiter vorangetrieben, was eine Verdoppelung der Betreuungsquote von Kindern unter drei Jahren von 16 auf 33 Prozent zwischen 2007 und 2017 brachte (Statistisches Bundesamt 2018: 117). Doch die Betreuungsdichte schwankt zwi- 
schen einzelnen Regionen stark. Vielerorts liegt die Anzahl verfügbarer Plätze unter der Nachfrage (Bundesministerium für Familie, Senioren, Frauen und Jugend 2018). Das hat zur Folge, dass der berufliche Wiedereinstieg der jungen Eltern - zumeist Mütter - aufgeschoben werden muss. Und so werden (wieder) verstärkt Betreuungsarrangements in der Familie und im privaten Umfeld gesucht. Das bezieht in erster Linie Großeltern ein, vor allem, wenn diese in der Nähe leben. Doch schränkt die in der späten Erwerbsphase angestiegene Frauenerwerbstätigkeit (Vogel \& Scherger 2018) gerade die Zeit- und Energieressourcen derer ein, die bislang typischerweise diese Sorgeaufgaben übernehmen, nämlich der Frauen.

\section{Geschlechterungleichheit bei der Ausübung unbezahlter Sorgetätigkeiten}

Frauen sind in deutlich größerem Umfang am Erbringen unbezahlter Sorgetätigkeiten beteiligt als Männer. Der für 2012/2013 über alle Altersgruppen hinweg ermittelte sogenannte Gender Care Gap liegt bei 52 Prozent (Deutscher Bundestag 2017: 95). Umgerechnet auf den täglichen Zeitaufwand bedeutet das, dass Frauen 87 Minuten mehr unbezahlte Sorgetätigkeiten leisten als Männer (ebd.: 96). Wie der Deutsche Alterssurvey eindrücklich belegt, sind es auch im mittleren und höheren Alter gerade die Frauen, die Leistungen dieser Art nicht nur häufiger, sondern auch zeitintensiver als Männer erbringen: Beispielsweise werden nach wie vor in einem Großteil der Partnerschaften ab dem 40. Lebensjahr anfallende Hausarbeiten überwiegend von den Frauen (61 Prozent) übernommen (Engstler \& Klaus 2017: 201). Auch die Enkelbetreuungs- und Unterstützungsquoten fallen unter 40- bis 85-jährigen Frauen statistisch signifikant höher aus (32 bzw. 19 Prozent) als unter den gleichaltrigen Männern (28 bzw. 13 Prozent) (Klaus \& Tesch-Römer 2017: 192; Mahne \& Klaus 2017: 242). Also auch für die zweite Lebenshälfte gilt: Haus-, Familien und Pflegearbeiten werden mehrheitlich von Frauen verantwortet.

\section{Unbezahlte Sorgetätigkeiten im Alternsverlauf}

Während Geschlechterunterschiede in der Übernahme von Sorgetätigkeiten gut belegt sind, liegen bislang kaum fundierte Informationen darüber vor, wie sich Sorgetätigkeiten über das mittlere bis ins hohe Erwachsenenalter hinweg verändern. Gibt es beispielsweise besonders beanspruchte Lebensphasen? Entsprechend wenig weiß man darüber, ob sich Frauen und Männer mit zunehmendem Alter und insbesondere nach Renteneintritt einander annähern oder nicht. Einzelne Studien und Statistiken legen nahe, dass ein Großteil der Aufgaben wie Enkelbetreuung oder Unterstützung älterer und unterstützungsbedürftiger Eltern in die späte Erwerbsphase fallen. Zwar ist das durchschnittliche Übergangsalter in die Großelternschaft in den letzten Jahren angestiegen, liegt aber mit 53 Jahren weiterhin im mittleren Erwachsenenalter (Mahne \& Klaus 2017: 236 und ähnliche und differenziertere Analysen bei Leopold \& Skopek 2015). Gut die Hälfte (53 Prozent) der privat Pflegeleistenden ist zwischen 40 und 64 Jahren alt (TNS Infratest Sozialforschung 2011: 27). Doch es wächst auch der Anteil hochaltriger Pflegepersonen (z. B. Runde, Giese, Kaphengst, \& Hess 2009; Rothgang, Iwansky, Müller, Sauer, \& Unger 2010). Überdies sind Empfängerinnen und Empfänger häufig bereits vor dem eintretenden Bedarf zeitintensiver Pflege auf Hilfe bei ihrer Alltagsbewältigung und Haushaltsorganisation angewiesen. Eine möglicherweise viele Jahre andauernde Unterstützungsbeziehung beginnt also nicht selten bereits in der Phase aktiver Erwerbstätigkeit der Unterstützungspersonen.

\section{Unbezahlte Sorgetätigkeiten und Erwerbstätigkeit}

Wie in frühen Lebens- und Familienphasen wird auch im mittleren und höheren Erwachsenenalter die stärkere Einbindung von Frauen in Hausund Sorgetätigkeiten von einer umfangreicheren 
Erwerbsbeteiligung der Männer begleitet. Doch selbst wenn die Gesamtarbeitszeit unbezahlter und bezahlter Arbeit von Frauen und Männern gleich ist (Hobler, Klenner, Pfahl, Sopp, \& Wagner 2017), impliziert diese geschlechtsspezifische Arbeitsteilung vielfältige Benachteiligungen für die Frauen. Offenkundig unterscheiden sich Erwerbsarbeit und unbezahlte Sorgetätigkeiten in ihrer monetären und damit auch gesellschaftlichen Gratifikation. Daraus resultiert nicht nur eine Ungleichverteilung sozialer Wertschätzung, sondern auch der finanziellen (Un-)Abhängigkeit und damit verbunden der Absicherung im Rentenalter. Die Übernahme von Kindererziehung und Pflegetätigkeiten in der Familie werden als Hauptrisikofaktoren für Armut im Alter genannt (Brettschneider \& Klammer 2016; Vogel \& Künemund 2018). Hinzu kommt - insbesondere für privat Pflegeleistende eine hohe gesundheitliche und soziale Belastung (Schwinger, Tsiasioti, \& Klauber 2016; Nowossadeck, Engstler, \& Klaus 2016; Engstler \& TeschRömer 2017; Bestmann, Wüstholz, \& Verheyen 2014). Sind Personen, die Fürsorgetätigkeiten leisten, zudem erwerbstätig, müssen beide Tätigkeitsbereiche miteinander vereinbart werden. Das geht oft mit zusätzlicher körperlicher sowie psychischer Beanspruchung der Sorgeleistenden einher. Nicht zu vergessen sind eingeschränkte Berufs- und Karrierechancen, wenn die Erwerbstätigkeit reduziert oder gar aufgegeben werden muss.

Das Spannungsverhältnis zwischen Erwerbsund Sorgetätigkeiten ist offenkundig. Es wurde vielfach untersucht und gesamtgesellschaftlich sowie politisch adressiert. Vor allem die Forschung zur Vereinbarkeit von Erwerbs- und Pflegetätigkeit hat gezeigt, dass sich die Übernahme von Pflege sowohl auf die Wahrscheinlichkeit, erwerbstätig zu bleiben als auch auf den Erwerbsumfang negativ auswirkt (u. a. Henz 2004; Bolin, Lindgren, \& Lundborg 2008; Keck 2012; Geyer 2016; Spiess \& Schneider 2003; Schäufele, Köhler, \& Hendlmeier 2016). So setzt nur die Hälfte (51 Prozent) der bei Pflegebeginn Erwerbstätigen ihre Erwerbstätigkeit in unverändertem Umfang fort, während 34 Prozent ihre Erwerbstätigkeit reduzieren und
15 Prozent sie ganz aufgegeben (TNS Infratest Sozialforschung 2011: 32). Dieser Effekt ist stärker bei Frauen als bei Männern (Frerk \& Leitner 2017: 276) und er ist nachhaltig: Ein beruflicher Wiedereinstieg nach der Pflegezeit ist unwahrscheinlich (Spiess \& Schneider 2003) und die Chancen hierfür sind gering (Keck 2016). Vergleichbare Untersuchungen zu Enkelbetreuung und informeller Unterstützung vor Pflegeeintritt gibt es kaum. Das mag daran liegen, dass hier keine Vereinbarkeitsproblematik vermutet wird. In der (gerontologischen) Familienforschung wird allenfalls - umgekehrt - der Einfluss von Erwerbstätigkeit auf die Übernahme dieser Sorgetätigkeiten untersucht. Die Befunde sind jedoch nicht eindeutig: Einigen Studien zufolge unterstützen und pflegen erwerbstätige erwachsene Kinder ihre Eltern seltener und weniger umfangreich als nicht erwerbstätige Kinder (Kalmijn \& Saraceno 2008; Leopold \& Raab 2011; Leopold, Raab, \& Engelhardt 2014). Erwerbstätige Großeltern betreuen ihre Enkel zwar nicht seltener, doch weniger umfangreich im Vergleich zu nicht erwerbstätigen Großeltern (Hank \& Buber 2009; Igel \& Szydlik 2011). In anderen Arbeiten erweist sich Erwerbstätigkeit in diesem Zusammenhang als unbedeutend (Deindl \& Brandt 2011; Klaus 2009: 235; Brandt, Haberkern, \& Szydlik 2009; Stuifbergen, van Delden, \& Dykstra 2008).

\section{Wandel in der Übernahme unbezahlter Sorgetätigkeiten}

Unbezahlte Sorgetätigkeiten haben im mittleren und höheren Lebensalter an Bedeutung gewonnen. Dabei hat sich die Nachfrage nach Betreuungsund Pflegeleistungen ebenso erhöht wie das Interesse, ältere Arbeitnehmerinnen und Arbeiternehmer so lang wie möglich im Beruf zu halten. Diese gesellschaftliche Entwicklung spiegelt sich auch in den individuellen Wünschen der Menschen wider: erwerbstätig zu sein und sich (zugleich) um unterstützungsbedürftige (Familien-)Angehörige kümmern zu können. Es ist anzunehmen, dass die gestiegene Bereitstellung und Organisation von 
Betreuung und Unterstützung im privaten Bereich durch die gestiegene Ressourcenausstattung der älteren Menschen begünstigt wird, die heute besser ausgebildet und gesünder sind als etwa noch vor 20 Jahren. Beispielsweise zeigen Untersuchungen, dass Menschen mit höherer Bildung anteilig häufiger Unterstützungsleistungen für Verwandte und nicht-verwandte Personen erbringen als Menschen mit geringer Bildung (Klaus \& Tesch-Römer 2017: 192; Vogel \& Tesch-Römer 2017: 263) und eine gute Gesundheit ist nachweislich Vorbedingung für das Leisten instrumenteller Hilfen wie Betreuung und Pflege. Allerdings sind Probleme der Vereinbarkeit von Erwerbs- und Sorgetätigkeiten ebenso bekannt wie die Tatsache, dass es häufiger Frauen sind, die vor dieser Aufgabe stehen. Weniger weiß man jedoch über die Dynamik der Geschlechterunterschiede bezüglich der Übernahme unbezahlter Sorgetätigkeiten im Verlauf des Älterwerdens sowie über einen möglichen gesellschaftlichen Wandel geschlechtsspezifischer Alternsverläufe über nachfolgende Geburtskohorten.

\subsection{Methoden}

\section{Stichprobe}

Zur Beantwortung der aufgeworfenen Fragestellungen werden die Daten des Deutschen Alterssurveys (DEAS) der Jahre 1996 bis 2017 verwendet. Im Verlauf der bislang sechs Erhebungswellen wurden 20129 Personen befragt, für die 39446 Beobachtungen vorliegen (zur Stichprobenziehung und Datenbasis siehe Kapitel 2 Design, Inhalte und Methodik des Deutschen Alterssurveys in diesem Band). Die Teilnahmebereitschaft in der Erstbefragung sowie die Wiederbefragungsquoten bewegen sich im Durchschnitt europäischer Studien. Doch da sowohl in der Erst- als auch in den Wiederholungsbefragungen Personen mit bestimmten Merkmalskombinationen jeweils unterschiedlich große Wahrscheinlichkeiten der (Wieder-)Teil-

\section{Fragestellungen}

Im vorliegenden Beitrag wird die Übernahme von unterschiedlichen Sorgetätigkeiten im Alternsverlauf dargestellt - getrennt für Frauen und Männer sowie im Kohortenvergleich. Im Fokus stehen zwei Sorgetätigkeiten, die typisch für die mittlere und hohe Lebensphase sind: die Betreuung von Enkelkindern sowie die Bereitstellung von Betreuung, Unterstützung oder Pflege für gesundheitlich eingeschränkte Personen. Die zwei Leitfragen lauten:

1) Bestehen aufgrund einer anhaltenden geschlechtsspezifischen Arbeitsteilung Unterschiede zwischen Frauen und Männern in der Übernahme von Sorgetätigkeiten im Verlauf der zweiten Lebenshälfte?

2) Deutet sich infolge des demografischen Wandels sowie der Veränderungen im Erwerbsverhalten der vergangenen Jahrzehnte eine Veränderung der geschlechtsspezifischen Muster und Verläufe in der Übernahme unbezahlter Sorgetätigkeiten an?

nahme haben, ist die verwendete Panelstichprobe nicht frei von Selektivität. Zwei Vorkehrungen wurden getroffen, um einen möglichen verzerrenden Einfluss der Selektivität auf Analysen und Schätzungen zu reduzieren: (1) Zum Ausgleich der geschichteten Stichprobenziehung in der Erstbefragung wird in allen Modellen neben dem Lebensalter, das die Zeitvariable darstellt, für $\mathrm{Ge}$ schlecht und Region (Ost- vs. Westdeutschland) kontrolliert. (2) Um die selektive Teilnahme in den Wiederbefragungen abzumildern, gehen auch die Untersuchungspersonen in die Schätzungen ein, die nur einmal an der Studie teilgenommen haben. 


\section{Indikatoren}

Über alle Befragungswellen hinweg wurden (nahezu) identische Fragen zur Übernahme von Enkelbetreuung sowie zur Übernahme von Pflege und Unterstützung für Andere gestellt. Die Kategorie „Andere“ umfasst sowohl Verwandte als auch nicht-verwandte Personen aus dem Freundes- und Bekanntenkreis sowie aus der Nachbarschaft. Entsprechend können vergleichbare Indikatoren erstellt werden. Für beide Sorgetätigkeiten werden dichotome Indikatoren gebildet. Diese geben an, ob zum Zeitpunkt der Befragung (1) Enkelkinder betreut werden (Wert 1 ) oder nicht (Wert 0 ) und ob (2) andere Personen auf Grund deren schlechten Gesundheitszustandes betreut, gepflegt oder anderweitig regelmäßig unterstützt werden (Wert 1) oder nicht (Wert 0). Dabei zeigt der Wert 0 entweder an, dass ein Enkelkind oder ein unterstützungsbedürftiger Angehöriger zwar vorhanden ist, aber nicht betreut wird. Oder der Wert 0 verweist darauf, dass es entsprechende potenzielle Empfängerinnen oder Empfänger nicht gibt, etwa weil die Befragungsperson keine Enkel hat. Somit beinhalten die Indikatoren eine sich möglicherweise geänderte Nachfrage nach diesen Sorgetätigkeiten, etwa weil in bestimmten Lebensphasen zunehmend mehr Personen (noch) keine Enkel haben. Im Falle der Pflege- und Unterstützungstätigkeit wird zusätzlich erfragt, wie viel Zeit für alle in diesem Sinne unterstützten Personen aufgewendet wird. Der hierzu generierte Indikator gibt den Wert in Wochenstunden an.

Um die Alternsverläufe der Ausübung dieser Tätigkeiten untersuchen und abbilden zu können, wird das Alter der Befragungsperson zum jeweiligen Befragungszeitpunkt berücksichtigt. Diese Variable geht als die zentrale Zeitvariable in die Analysen ein. Dabei kann sich die Wahrscheinlichkeit von Sorgetätigkeiten linear mit zunehmendem Alter verändern, aber auch nicht-linear verlaufen: etwa in Form einer Kurve, wenn ein Anstieg im frühen mittleren Erwachsenenalter im hohen Alter in einen Rückgang übergeht. Um das zu prüfen, geht das Alter sowohl in linearer als auch in quadrierter Form in die Modellschätzungen ein.

Um festzustellen, ob sich die Alternseffekte für Frauen und Männer unterscheiden, wird das $G e$ schlecht der Befragungsperson berücksichtigt. $\mathrm{Ob}$ mögliche Geschlechterunterschiede einem gesellschaftlichen Wandel unterliegen oder nicht, wird über die Aufnahme der Kohortenzugehörigkeit getestet. Es werden drei zentrale Geburtskohorten berücksichtigt, für die über eine größtmögliche Altersspanne empirische Beobachtungen (Messungen) vorliegen: zwischen 1930 und 1939 Geborene, von 1940 bis 1949 Geborene und von 1950 bis 1959 Geborene.

\section{Statistisches Analyseverfahren}

Die Schätzung der Alternsverläufe erfolgt anhand von Random-Effects-Panelregressionen (z. B. Brüderl 2010; Schröder 2007). Dieses Analyseverfahren nutzt alle vorliegenden Datenpunkte zwischen 1996 und 2017, das heißt alle Beobachtungen aller Studienteilnehmerinnen und -teilnehmer. Anders als bei Regressionen, die allein auf Querschnittdaten basieren (d.h. einmalige Befragung von Personen) geht demnach auch die Entwicklung (Stabilität oder Veränderung) der mehrfach befragten Personen ab dem Alter 40 in die Schätzung ein. Deshalb sind Panelregressionsmodelle verlässlicher und effizienter als querschnittliche Schätzmodelle, wenn es um die Schätzung von Alternsverläufen in den interessierenden Lebensbereichen geht (hier über die abgebildeten Alternsverläufe illustriert).

Für die beiden dichotom (das heißt in Form von ja-/nein-Angaben) vorliegenden Indikatoren der Sorgetätigkeit werden logistische Panelregressionen berechnet. Diese schätzen die Wahrscheinlichkeit, mit der die eine oder andere Sorgetätigkeit über den Alternsverlauf hinweg ausgeübt wird. Dabei wird nicht nur geprüft, ob die Ausübung einer Sorgetätigkeit sich überhaupt mit dem Alter verändert, sondern auch, welche Form der Alternsverlauf annimmt. Die auf Basis der Panelregression 
geschätzten Wahrscheinlichkeiten der Sorgetätigkeit werden grafisch dargestellt.

Um festzustellen, ob sich Frauen und Männer unterscheiden, wird Geschlecht als Variable aufgenommen. In den Schätzungen werden jeweils zwei Prüfungen vorgenommen: (1) Erstens wird getestet, ob sich Frauen und Männer hinsichtlich ihres Niveaus der Ausübung der Sorgetätigkeiten unterscheiden. Dabei wird die Signifikanzprüfung durchweg für das 60. Lebensjahr vorgenommen. Dieses Alter liegt etwas unter dem mittleren Alter der Gesamtstichprobe und zudem in der inhaltlich bedeutsamen späten Erwerbsphase. (2) Zweitens wird geprüft, ob sich die Verläufe zwischen den Geschlechtern unterscheiden. Das kann sich beispielsweise an unterschiedlich starken Anstie-

\subsection{Ergebnisse}

\subsubsection{Enkelbetreuung im Verlauf der zweiten Lebenshälfte}

Frauen betreuen Enkelkinder früher im Verlauf der zweiten Lebenshälfte sowie im Alter von 60 Jahren mit höherer Wahrscheinlichkeit als Männer.

Die Abbildung 6-1 zeigt die Enkelbetreuung im Verlauf des Älterwerdens. Dabei wird ein umgekehrt u-förmiger Verlauf zwischen dem 40. und dem 90. Lebensjahr erkennbar: Die Enkelbetreuung kommt zu Beginn und zum Ende der betrachteten Altersspanne praktisch nicht vor. Die Betreuungswahrscheinlichkeit liegt hier bei jeweils null Prozent. Sie häuft sich im 7. Lebensjahrzehnt. Ihren Höchstwert erreicht sie im Alter von 65 Jahren: Wenn Personen dieses Lebensalter erreichen, dann liegt den Schätzungen zufolge die durchschnittliche Wahrscheinlichkeit, mindestens ein Enkelkind zu betreuen, bei 24 Prozent.

Doch das gilt nicht gleichermaßen für Frauen und Männer: Im Alter von 60 Jahren geht die Wahrscheinlichkeit der Enkelbetreuung um zehn Prozentpunkte auseinander (Frauen: 26 Prozent, gen zeigen oder auch darin, dass die durchschnittliche Wahrscheinlichkeit der Übernahme von Sorgetätigkeiten in jeweils unterschiedlichem Alter ihr Maximum erreicht. Hierzu wird geprüft, ob die Interaktion zwischen Alter und Geschlecht statistisch signifikant ist. Mögliche Geschlechterunterschiede werden zudem für die drei ausgewählten Geburtskohorten vergleichend betrachtet, um zu prüfen, ob sich diese Geschlechterunterschiede in den Alternsverläufen verändert haben. Das erfolgt über eine zusätzliche Interaktion von Geschlecht und Alter mit der Geburtskohortenzugehörigkeit. Zur Ermittlung der statistischen Signifikanz wird in allen Analysen das Signifikanzniveau auf fünf Prozent $(\mathrm{p}<0,05)$ gesetzt.

Männer: 16 Prozent). Ihren Höhepunkt erreicht diese Diskrepanz bereits im 56. Lebensjahr: Hier unterscheidet sich die Wahrscheinlichkeit von Enkelbetreuung zwischen den Geschlechtern um elf Prozentpunkte. Spätestens mit Beginn des Beobachtungszeitraumes setzt eine Spreizung der Verläufe ein: Während die Wahrscheinlichkeit bei den Frauen schon ab dem 40. Lebensjahr sichtbar ansteigt, setzt eine vergleichbar starke Zunahme bei den Männern erst ca. fünf Jahre später ein. Zudem betreuen Frauen früher im Verlauf ihrer zweiten Lebenshälfte als Männer. Ihren Maximalwert erreichen Frauen mit 63 Jahren (27 Prozent), Männer mit 67 Jahren (21 Prozent). Die nach dem 56. Lebensjahr einsetzende Angleichung der Alternsverläufe ist mit Anfang 70 erreicht: Danach unterscheiden sich die Geschlechter in der Wahrscheinlichkeit von Enkelkinderbetreuung statistisch nicht mehr signifikant.

Enkelkinderbetreuung wird von nachfolgenden Kohorten in zunehmend höherem Alter übernommen. 
Abbildung 6-1 Wahrscheinlichkeit der Enkelbetreuung bei Frauen und Männern im Alternsverlauf

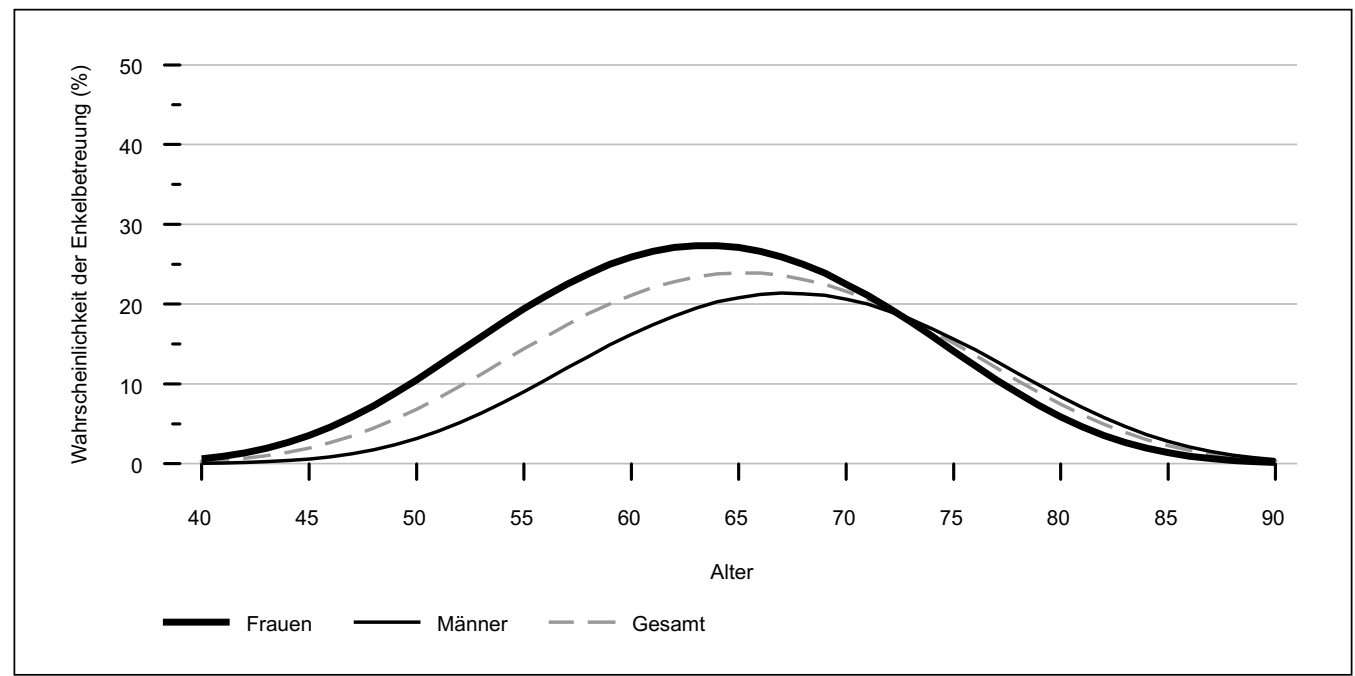

Quelle: DEAS 1996-2017, Schätzungen basieren auf n = 20114 Studienteilnehmenden mit insgesamt $\mathrm{n}=39361$ Beobachtungen, kontrolliert für Region.

Bei Frauen steigt die Wahrscheinlichkeit, Enkelkinder zu betreuen auf ein Maximum von 27 Prozent an (mit 63 Jahren), um danach wieder zu sinken. Bei Männern steigt die Wahrscheinlichkeit auf ein Maximum von 21 Prozent an (mit 67 Jahren). Im Alter von 90 Jahren liegt die Wahrscheinlichkeit der Enkelkinderbetreuung sowohl bei Frauen als auch bei Männern bei null. Der Unterschied zwischen Frauen und Männern in der Wahrscheinlichkeit im Alter von 6o Jahren ist signifikant. Ebenso unterscheidet sich der geschätzte Verlauf der Wahrscheinlichkeit der Enkelbetreuung zwischen Frauen und Männern signifikant.

Die Übernahme der Enkelbetreuung im Alternsverlauf hat sich über die betrachteten Geburtskohorten hinweg signifikant verändert; vor allem findet sie vermehrt in deutlich höherem Alter statt (Abbildung 6-2). Die Wahrscheinlichkeit, im Alter von 60 Jahren mindestens ein Enkelkind zu betreuen, sinkt von 25 Prozent in der Kohorte der zwischen 1930 und 1939 Geborenen, über 23 Prozent in der mittleren Geburtskohorte auf 20 Prozent in der Kohorte der zwischen 1950 und 1959 Geborenen. Die maximale Betreuungswahrscheinlichkeit wird in zunehmend höherem Alter erreicht: In der Kohorte der in den 1930er Jahren Geborenen im Lebensalter von 60 Jahren (25 Prozent), in der mittleren Geburtskohorte im Lebensalter von 66 Jahren (25 Prozent). In der Kohorte der zwischen 1950 und 1959 Geborenen liegt sie vermutlich sogar außerhalb des Beobachtungszeitraumes: Unter der Annahme, dass sich der Kohor- tenwandel so fortsetzt wie auf Grundlage der vorliegenden Daten geschätzt, erreicht diese Kohorte ihre höchste Betreuungswahrscheinlichkeit im Alter von 70 Jahren: mit 28 Prozent (diese voraussichtliche zukünftige Entwicklung ist nicht abgebildet).

Frauen und Männer betreuen Enkelkinder mit zunehmend gleicher Wahrscheinlichkeit.

Eine nach Geburtskohorten getrennte Betrachtung legt einen Rückgang der Geschlechterunterschiede bei aufeinander folgenden Kohorten nahe (Abbildung 6-3). Diese Angleichung über die Kohorten hinweg zeigt sich in der Wahrscheinlichkeit der Enkelbetreuung: In der Kohorte der in den 1930er Jahren Geborenen fällt der Geschlechterunterschied im Alter von 60 Jahren mit 15 Prozentpunkten am größten aus. In beiden Nachfolgekohorten 
Abbildung 6-2 Wahrscheinlichkeit der Enkelbetreuung nach Kohorten im Alternsverlauf

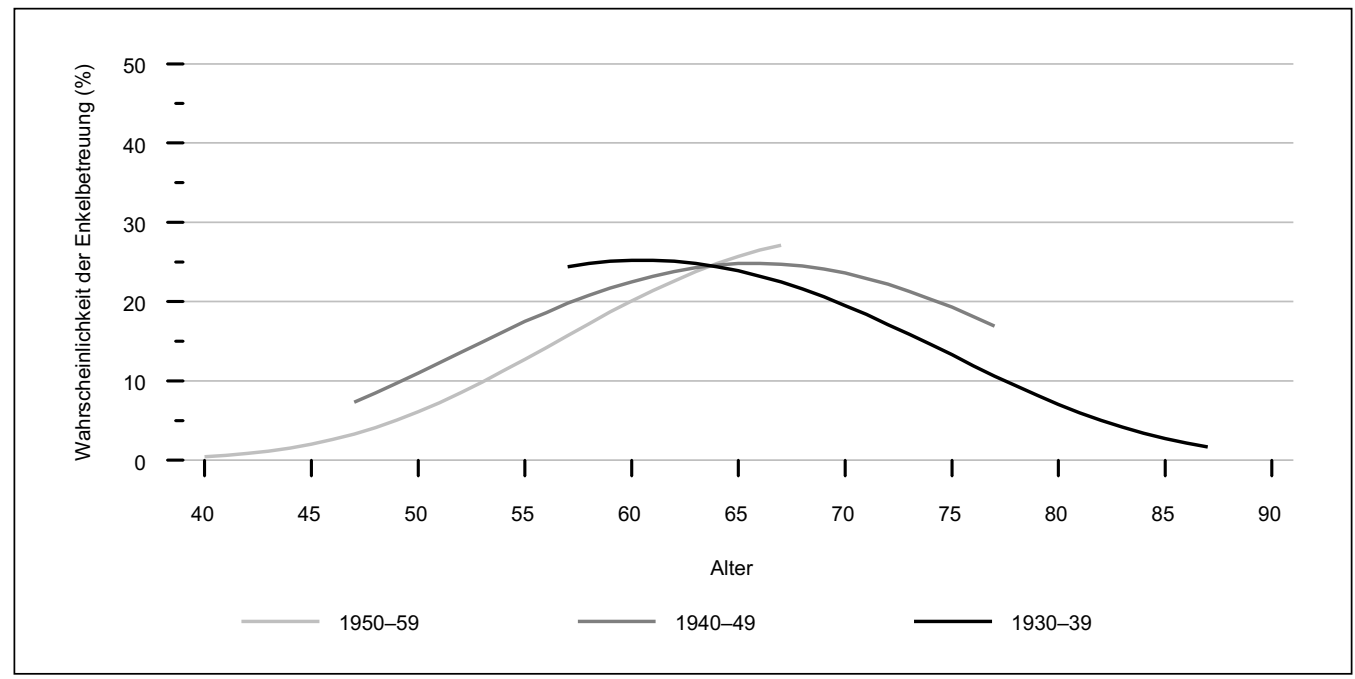

Quelle: DEAS 1996-2017, Schätzungen beruhen auf $n=13995$ Studienteilnehmenden mit insgesamt $n=29198$ Beobachtungen, kontrolliert für Region und Geschlecht.

Für die Kohorte der 1930 bis 1939 Geborenen steigt die Wahrscheinlichkeit der Enkelbetreuung bis zum Alter von 6o Jahren an (25 Prozent). Für die zwischen 1940 und 1949 geborenen Personen ist bis zum Alter von 66 Jahren (25 Prozent) ein Anstieg zu beobachten. Für die Kohorte der 1950 bis 1959 Geborenen ist ein Anstieg der Wahrscheinlichkeit der Enkelbetreuung bis zum Alter von 67 Jahren (27 Prozent) zu beobachten. Der Unterschied zwischen den Geburtskohorten in der durchschnittlichen Wahrscheinlichkeit der Übernahme von Enkelkinderbetreuung im Alter von 6o Jahren ist signifikant. Ebenso unterscheidet sich der geschätzte Verlauf der Wahrscheinlichkeit der Enkelbetreuung zwischen den Kohorten signifikant.

ist er jeweils kleiner: elf Prozentpunkte bei den in den 1940er Jahren Geborenen und acht Prozentpunkte bei den in den 1950er Jahren Geborenen. Hinter der Annäherung des Betreuungsverhaltens über die Kohorten hinweg stehen unterschiedliche Veränderungen bei Frauen und Männern. Zwar zeigt sich bei beiden Geschlechtern im Alter von 60 Jahren eine Abnahme der Betreuungswahrscheinlichkeit über nachfolgende Kohorten. Doch fällt dieser Rückgang bei den Frauen deutlich größer aus (von 33 Prozent in der Kohorte der 1930 bis 1939 Geborenen über 28 Prozent auf 24 Prozent in der Kohorte der 1950 bis 1959 Geborenen) als bei den Männern (von 18 Prozent in der Kohorte der 1930 bis 1939 Geborenen über 17 Prozent auf 16 Prozent in der Kohorte der 1950 bis 1959 Geborenen). Ein Vergleich der Maximalwerte belegt ebenfalls, dass Frauen - im Vergleich zu Männern - zunehmend mit geringerer Wahr- scheinlichkeit Enkelkinder betreuen: Bei ihnen sinkt der Maximalwert von 34 Prozent (Kohorte 1930-1939) auf 29 Prozent (Kohorte 1950-1959). Männer der später geborenen Geburtskohorten betreuen sogar mit etwas größerer Wahrscheinlichkeit als ihre Vorgänger: Ihr Maximalwert steigt in der Kohortenabfolge von 20 Prozent auf 28 Prozent.

Die Geschlechterdiskrepanz im Alternsverlauf bleibt hingegen unverändert. Hier kommt es zu keiner Annäherung zwischen Frauen und Männern. Zwar werden Enkel in einem zunehmend höheren Alter betreut, allerdings findet diese Entwicklung für beide Geschlechter gleichermaßen statt. Sowohl Frauen als auch Männern jüngerer Geburtskohorten betreuen später in der zweiten Lebenshälfte: Lag das Maximum in der Kohorte der in den 1930er geborenen Frauen bei 56 Jahren, so stieg es in der Kohorte der 1940 bis 1949 gebo- 
Abbildung 6-3 Wahrscheinlichkeit der Enkelbetreuung bei Frauen und Männern nach Kohorten im Alternsverlauf

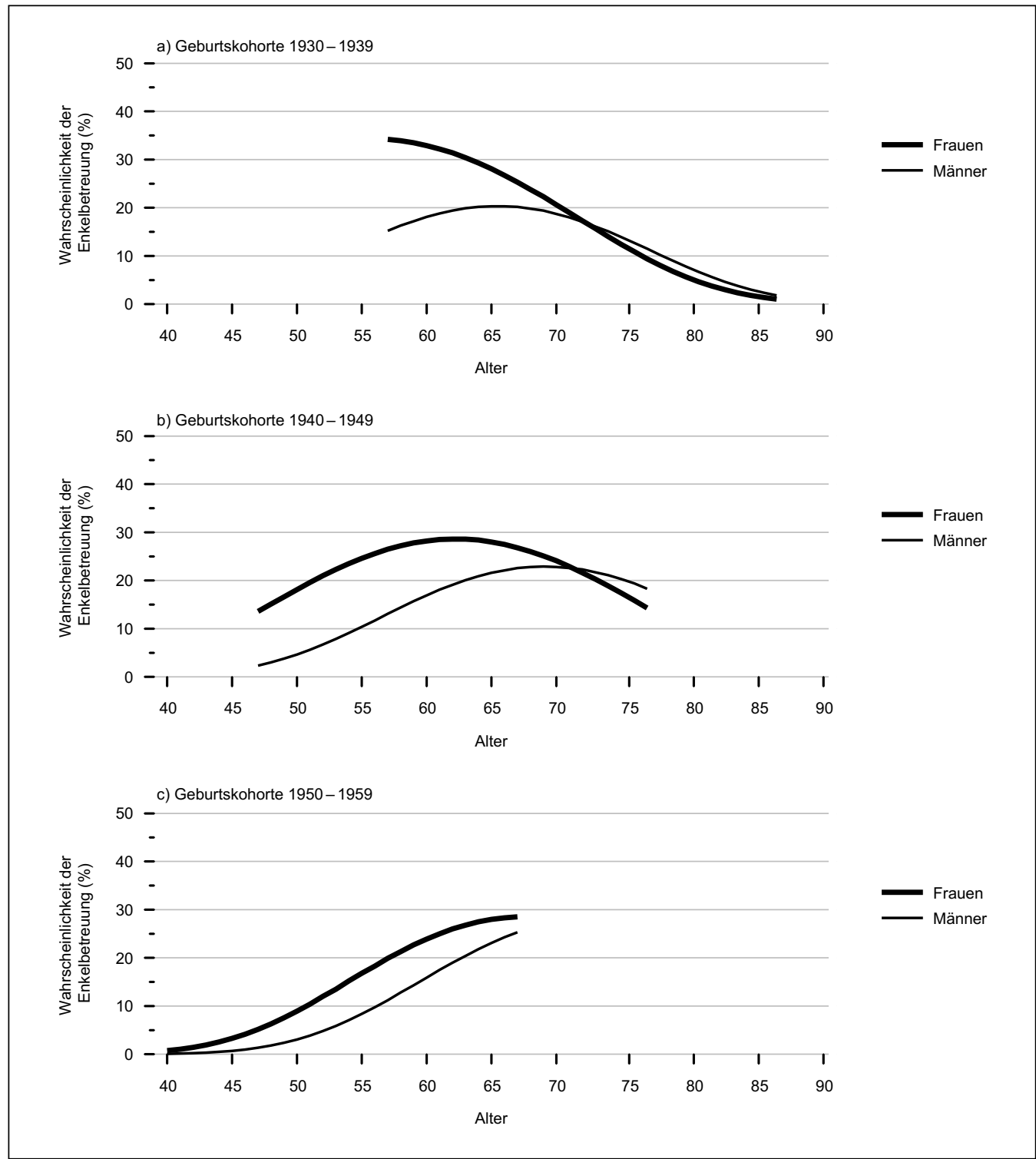

Quelle: DEAS 1996-2017, Schätzungen basieren auf n = 13995 Studienteilnehmenden mit insgesamt n = 29198 Beobachtungen, kontrolliert für Region.

In der Kohorte der 1930 bis 1939 Geborenen liegt die Wahrscheinlichkeit der Enkelbetreuung im Alter von 6o Jahren bei 33 Prozent bei den Frauen und bei 18 Prozent bei den Männern. Dieser Geschlechterunterschied reduziert sich über die nachfolgenden Kohorten hinweg signifikant: In der Kohorte der 1950 bis 1959 Geborenen beträgt die Wahrscheinlichkeit der Enkelbetreuung im Alter von 6o Jahren 24 Prozent bei den Frauen und 16 Prozent bei den Männern. Der geschätzte geschlechtsspezifische Verlauf der Wahrscheinlichkeit der Enkelbetreuung verändert sich über die Kohorten hinweg nicht signifikant: Geschlechterunterschiede im Alternsverlauf zeigen sich in allen drei betrachteten Kohorten gleichermaßen. 
renen Frauen auf 62 Jahre und in der Kohorte der 1950 bis 1959 geborenen Frauen lag es bereits bei 67 Jahren. In ähnlichem Umfang verläuft die Verlagerung bei den Männern: In der Kohorte 19301939 erreichen sie ihren Maximalwert mit 65 Jahren; in der Kohorte 1940-1949 mit 69 Jahren und in der jüngsten Kohorte 1950-1959 erst mit 73 Jahren. Damit bleibt der Geschlechterunterschied im Verlauf über die Kohorten hinweg erhalten. Das heißt, auch in der jüngsten Geburtskohorte betreuen Frauen deutlich früher als Männer.

\subsubsection{Unterstützung und Pflege im Verlauf der zweiten Lebenshälfte}

Frauen leisten Unterstützung und Pflege früher im Verlauf der zweiten Lebenshälfte als Männer sowie im Alter von 60 Jahren mit höherer Wahrscheinlichkeit.

Anders als die Enkelbetreuung, die sich auf eine bestimmte Lebensphase der älteren Personen konzentriert, verteilt sich die Unterstützung und Pflege von Angehörigen stärker über die gesamte zweite Lebenshälfte (Abbildung 6-4). Das gilt mehr noch für Männer als für Frauen. Doch auch in diesem Bereich der Sorgetätigkeiten kommt es im Verlauf des Älterwerdens zunächst zu einem Anstieg, der noch vor dem Erreichen des offiziellen Rentenalters in einen Rückgang übergeht. Die Betrachtung über beide Geschlechter hinweg zeigt, dass im Alter von 40 Jahren die Wahrscheinlichkeit, gesundheitlich eingeschränkte Angehörige zu unterstützen, zu betreuen und/oder zu pflegen bei zehn Prozent liegt. Im weiteren Alternsverlauf gewinnen diese Tätigkeiten an Bedeutung, um sich im Lebensalter von Mitte 50 bis Mitte $60 \mathrm{zu}$ häufen: Zwischen dem 54. und dem 65. Lebensjahr bewegt sich die Wahrscheinlichkeit auf einem Plateau von 16 Prozent. Danach setzt ein Rückgang ein: Dieser verläuft weniger steil als bei der Enkelbetreuung und auch mit 90 Jahren liegt die Wahrscheinlichkeit zu unterstützen und/oder zu pflegen noch bei fünf Prozent.
In Analogie zur Enkelbetreuung zeigen sich auch hier deutliche Geschlechterunterschiede sowohl im Niveau als auch im Verlauf des Älterwerdens: Männer leisten im Alter von 60 Jahren durchschnittlich seltener Unterstützung und Pflege für Verwandte und Personen aus dem Freundes- und Bekanntenkreis oder der Nachbarschaft als dies Frauen tun. Die Wahrscheinlichkeit liegt bei Frauen, wenn sie das 60. Lebensjahr erreichen, bei 20 Prozent; bei Männern bei 13 Prozent. Im höheren Alter kommt es zu einer allmählichen Angleichung: Ab dem 77. Lebensjahr zeigen sich in der Wahrscheinlichkeit des Unterstützungsverhaltens keine signifikanten Unterschiede mehr zwischen Frauen und Männern. Abgesehen von dieser Geschlechterdiskrepanz unterscheiden sich auch die Alternsverläufe. So nimmt die Wahrscheinlichkeit für Unterstützung und Pflege im Verlauf der späten Erwerbsphase bei Frauen deutlich zu: zwischen dem 40. und dem 57. Lebensjahr von zwölf Prozent auf 20 Prozent (Maximum). Danach nimmt die Wahrscheinlichkeit erheblich ab: auf drei Prozent im Alter von 90 Jahren. Anders sieht es für die Männer aus, für die Anstieg und Rückgang deutlich milder ausgeprägt sind: Im Alter von 40 liegt ihre Wahrscheinlichkeit bei acht Prozent, erreicht mit 61 Jahren ihren Höhepunkt (13 Prozent) und geht bis zum Alter von 90 Jahren auf sechs Prozent zurück. Die Kurve entlang des Älterwerdens fällt vergleichsweise flach aus. Dieser Sorgetätigkeit gehen Männer also nicht nur seltener nach, ihre Übernahmewahrscheinlichkeit verändert sich auch weniger mit dem Alter. Anders ist es bei den Frauen: Für sie stellt sich die späte Erwerbsphase als besonders beanspruchte Lebensphase heraus.

In später geborenen Kohorten wird Unterstützung und Pflege zunehmend früher im Verlauf der zweiten Lebenshälfte geleistet und ihre Wahrscheinlichkeit im Alter von 60 Jahren nimmt $z u$.

Unabhängig vom Geschlecht nimmt über die betrachteten Geburtskohorten hinweg die Bereitstellung von Unterstützung und Pflege für kranke 
Abbildung 6-4 Wahrscheinlichkeit gesundheitsbedingter Unterstützung und Pflege bei Frauen und Männern im Alternsverlauf

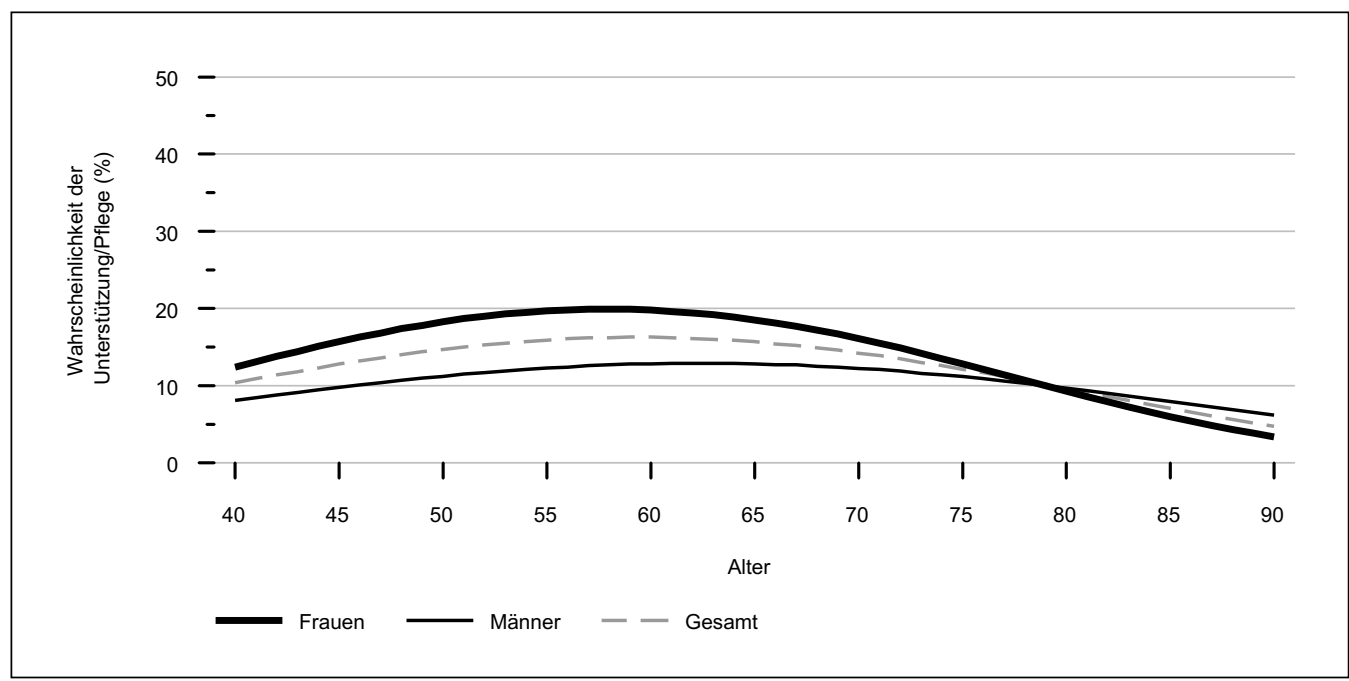

Quelle: DEAS 1996-2017, Schätzungen basieren auf $n=20114$ Studienteilnehmenden mit insgesamt n = 39342 Beobachtungen, kontrolliert für Region.

Bei Frauen steigt die Wahrscheinlichkeit, Angehörige zu unterstützen und/oder zu pflegen von zwölf Prozent (mit 40 Jahren), auf 20 Prozent (mit 57 Jahren) und geht danach zurück auf drei Prozent (mit 90 Jahren). Bei den Männern steigt die Wahrscheinlichkeit von acht Prozent (mit 40 Jahren) auf ${ }_{13}$ Prozent (mit 61 Jahren). Nach diesem Höchstwert geht sie auf sechs Prozent zurück (mit 90 Jahren). Sowohl der Unterschied in der Wahrscheinlichkeit im Alter von 6o Jahren als auch im geschätzten Alternsverlauf zwischen Frauen und Männern sind signifikant.

Angehörige zu (Abbildung 6-5). Diese Unterstützungsleistung wird im Alter von 60 Jahren mit einer Wahrscheinlichkeit von zwölf Prozent bei den in den 1930ern Geborenen übernommen; die Wahrscheinlichkeit beträgt 16 Prozent bei den in den 1940ern Geborenen und 19 Prozent bei den in den 1950er Jahren Geborenen.

Doch nicht nur die Wahrscheinlichkeit dieser Sorgetätigkeit ist gestiegen, auch der Alternsverlauf hat sich über die Kohorten hinweg stark verändert: Der umgekehrt u-förmige Verlauf, der in der Kohorte der in den 1950er Jahren Geborenen deutlich ausgeprägt ist, ist signifikant verschieden vom Verlauf der Kohorte der 1930 bis 1939 Geborenen. So erreicht die Unterstützungswahrscheinlichkeit bei den zwischen 1950 und 1959 Geborenen im Alter von 61 Jahren ihren höchsten Wert (19 Prozent). In diesem Alter bewegt sich die
Wahrscheinlichkeit der 1930er Geburtskohorte auf ihren Tiefstwert zu, den sie im Alter von 69 Jahren mit elf Prozent erreicht. Zugleich zeigt sich für diese Geburtskohorte der 1930 bis 1939 Geborenen eine vergleichsweise geringere Veränderung mit dem Alter: Unterstützung und/oder Pflege für gesundheitlich eingeschränkte Angehörige werden hier in jedem Alter der zweiten Lebenshälfte mit etwa gleich großer Wahrscheinlichkeit erbracht. In der Kohorte der 1950 bis 1959 Geborenen hingegen konzentrieren sich diese Sorgetätigkeiten vor allem auf das Alter um die 60, um danach deutlicher zurückzugehen als noch in der vorangehenden Kohorte der 1940 bis 1949 Geborenen.

Geschlechtsspezifische Unterschiede im Bereich Unterstützung und Pflege bleiben über nachfolgende Geburtskohorten unverändert. 
Abbildung 6-5 Wahrscheinlichkeit gesundheitsbedingter Unterstützung und Pflege nach Kohorten im Alternsverlauf

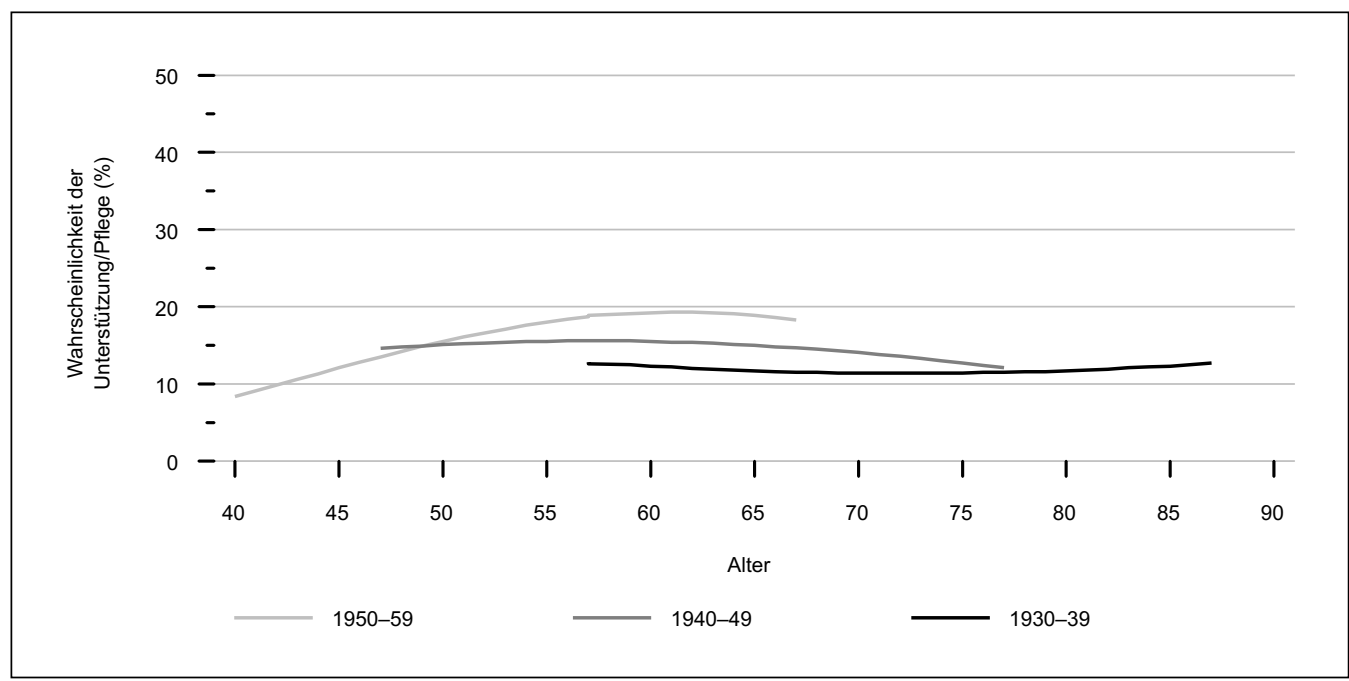

Quelle: DEAS 1996-2017, Schätzungen basieren auf n = 13998 Studienteilnehmenden mit insgesamt n = 29186 Beobachtungen, kontrolliert für Region und Geschlecht.

Für die Kohorte der 1930 bis 1939 Geborenen zeigt sich im Beobachtungszeitraum ein leichter Rückgang der Wahrscheinlichkeit Unterstützung und/oder Pflege zu leisten von 13 Prozent (im Alter von 57 Jahren) auf elf Prozent (im Alter von 69 Jahren), um danach wieder auf 13 Prozent anzusteigen (im Alter von 87 Jahren). Für die zwischen 1940 und 1949 geborenen Personen ist ein Anstieg von 15 Prozent (im Alter von 47 Jahren) auf 16 Prozent (im Alter von 56 Jahren) zu beobachten, sowie ein anschließender Rückgang auf zwölf Prozent (im Alter von 77 Jahren). Für die jüngste Kohorte (1950 bis 1959) ist ein Anstieg der Wahrscheinlichkeit von acht Prozent (im Alter von 40 Jahren) auf 19 Prozent (im Alter von 61 Jahren) zu beobachten. Bis zum Ende des Beobachtungszeitraums für diese Kohorte (im Alter von 67 Jahren) geht dieser Wert auf 18 Prozent zurück. Sowohl Unterschiede in der Wahrscheinlichkeit im Alter von 6o Jahren wie Unterschiede im geschätzten Verlauf der Wahrscheinlichkeit erweisen sich über die betrachteten Kohorten hinweg als signifikant.

Trotz des gesellschaftlichen Wandels in der Unterstützungs- und Pflegetätigkeit gibt es keine Hinweise, dass sich damit auch die geschlechtsspezifischen Muster verändern würden (Abbildung 6-6): Über alle drei Geburtskohorten hinweg bleiben die Unterschiede zwischen Frauen und Männern erhalten: Die Alternsverläufe beider Geschlechter verlaufen ungefähr parallel zueinander mit einer durchweg höheren Wahrscheinlichkeit für Frauen, Verwandte oder nicht-verwandte Personen zu unterstützen und/oder zu pflegen. Demnach zeichnet sich in diesem Bereich der Sorgetätigkeiten, im Gegensatz zur Enkelbetreuung, bislang keine Annäherung der Geschlechter ab.
Der zeitliche Umfang geleisteter Unterstützungsund Pflegeleistungen steigt im Verlauf der zweiten Lebenshälfte an.

Eine ergänzende Betrachtung des zeitlichen Umfangs der geleisteten Unterstützung und Pflege legt offen, dass sich die Intensität der erbrachten Leistungen umgekehrt $\mathrm{zu}$ ihrer Wahrscheinlichkeit entwickelt (Abbildung 6-7): In dem Alter, in dem diese Sorgetätigkeit am häufigsten übernommen wird, fällt ihr Umfang am geringsten aus. Bis Ende 50 - das Alter also, in der die Unterstützungswahrscheinlichkeit ihren Höhenpunkt erreicht - bewegt sich die Intensität auf ihrem geringsten Niveau von kaum mehr als zehn Stunden pro Woche. Von da 
Abbildung 6-6 Wahrscheinlichkeit gesundheitsbedingter Unterstützung und Pflege bei Frauen und Männern nach Kohorten im Alternsverlauf

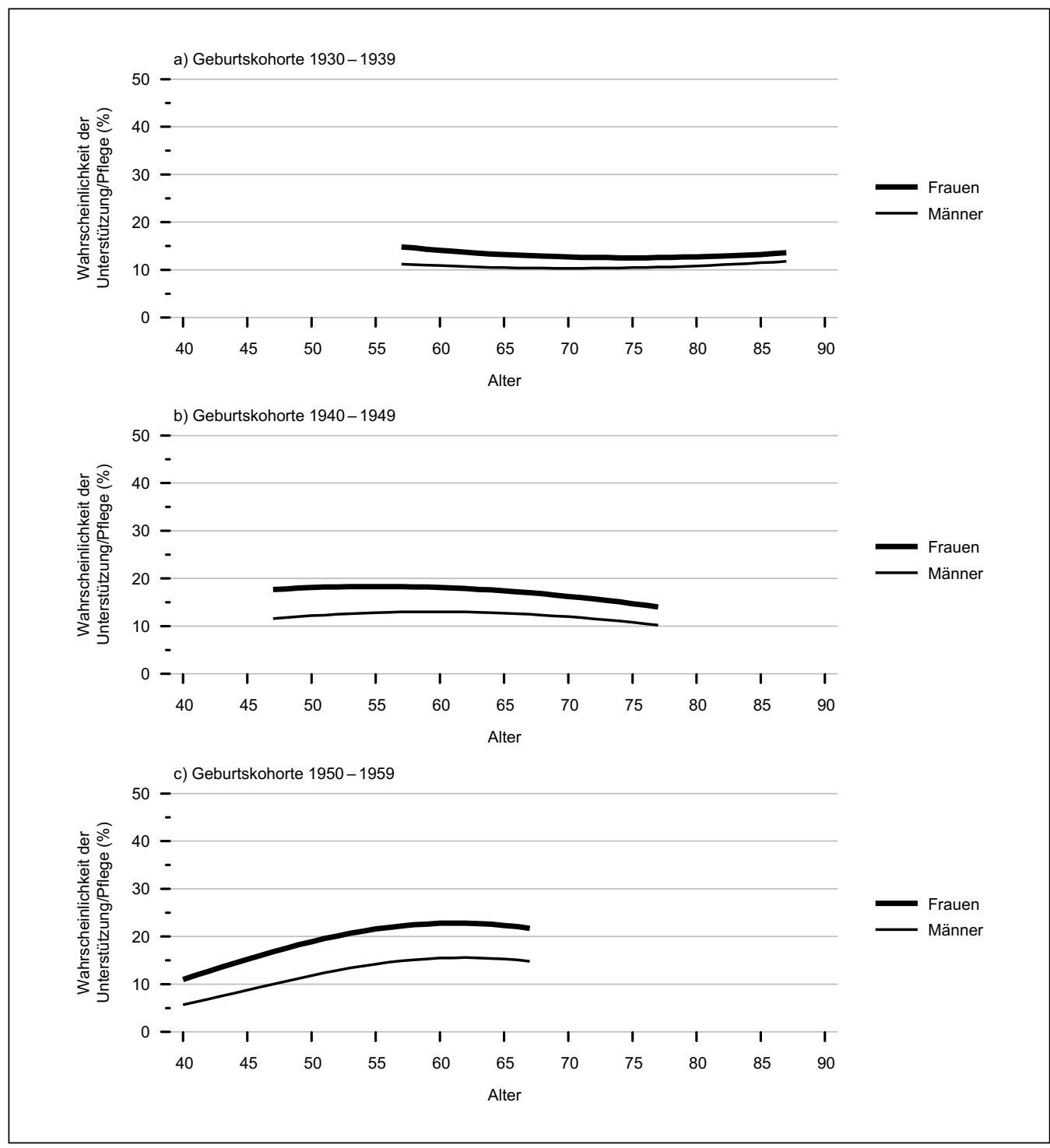

Quelle: DEAS 1996-2017, Schätzungen basieren auf $n=13998$ Studienteilnehmenden mit insgesamt $n=29186$ Beobachtungen, kontrolliert für Region.

In der Kohorte der 1930 bis 1939 Geborenen liegt die Wahrscheinlichkeit, Unterstützung und/oder Pflege zu leisten, im Alter von 60 Jahren bei 14 Prozent bei den Frauen und bei elf Prozent bei den Männern. In der mittleren Kohorte (1940 bis 1949) beträgt die vergleichbare Wahrscheinlichkeit 18 Prozent bei den Frauen und ${ }_{13}$ Prozent bei den Männern. In der Kohorte der 1950 bis 1959 Geborenen beträgt sie 23 Prozent bei den Frauen und 16 Prozent bei den Männern. Die geschätzten Geschlechterunterschiede der Wahrscheinlichkeiten ändern sich weder in ihrem Niveau noch im Alternsverlauf über die Kohorten hinweg. 
an beginnt sie jedoch sichtlich anzuwachsen und erreicht im Alter von 79 Jahren einen doppelt so hohen Umfang: In diesem Alter üben Personen, die mindestens eine andere Person unterstützen und/oder pflegen, diese Tätigkeit im Durchschnitt 20 Wochenstunden aus. Danach steigt sie weiter über den betrachteten Zeitraum auf 27 Stunden im Alter von 90 Jahren.

Dabei fällt der Anstieg im Alternsverlauf bei den Männern deutlicher aus als bei den Frauen (Abbildung 6-7). Allerdings erweisen sich diese Geschlechterunterschiede nur in der Altersphase als statistisch bedeutsam, in der diese Tätigkeit am häufigsten übernommen wird: zwischen dem 47. und 66. Lebensjahr. Für diese Altersspanne zeigt sich also nicht nur, dass Frauen dieser Sorgetätigkeit häufiger nachgehen, sondern dies auch mit größerer Intensität tun: Im Alter von 60 Jahren übernehmen Frauen, die mindestens eine angehörige Person aufgrund deren schlechter $\mathrm{Ge}$ sundheit unterstützen, betreuen und/oder pflegen, diese Aufgabe durchschnittlich 13 Wochenstunden. Männer, die diese Sorgetätigkeit ausüben, tun das in diesem Alter im Umfang von zehn Stunden pro Woche. Der geschlechtsspezifische Alternsverlauf zeigt zunächst ein Anwachsen des Geschlechter-

Abbildung 6-7 Umfang gesundheitsbedingter Unterstützung und Pflege (in Wochenstunden) bei Frauen und Männern im Alternsverlauf

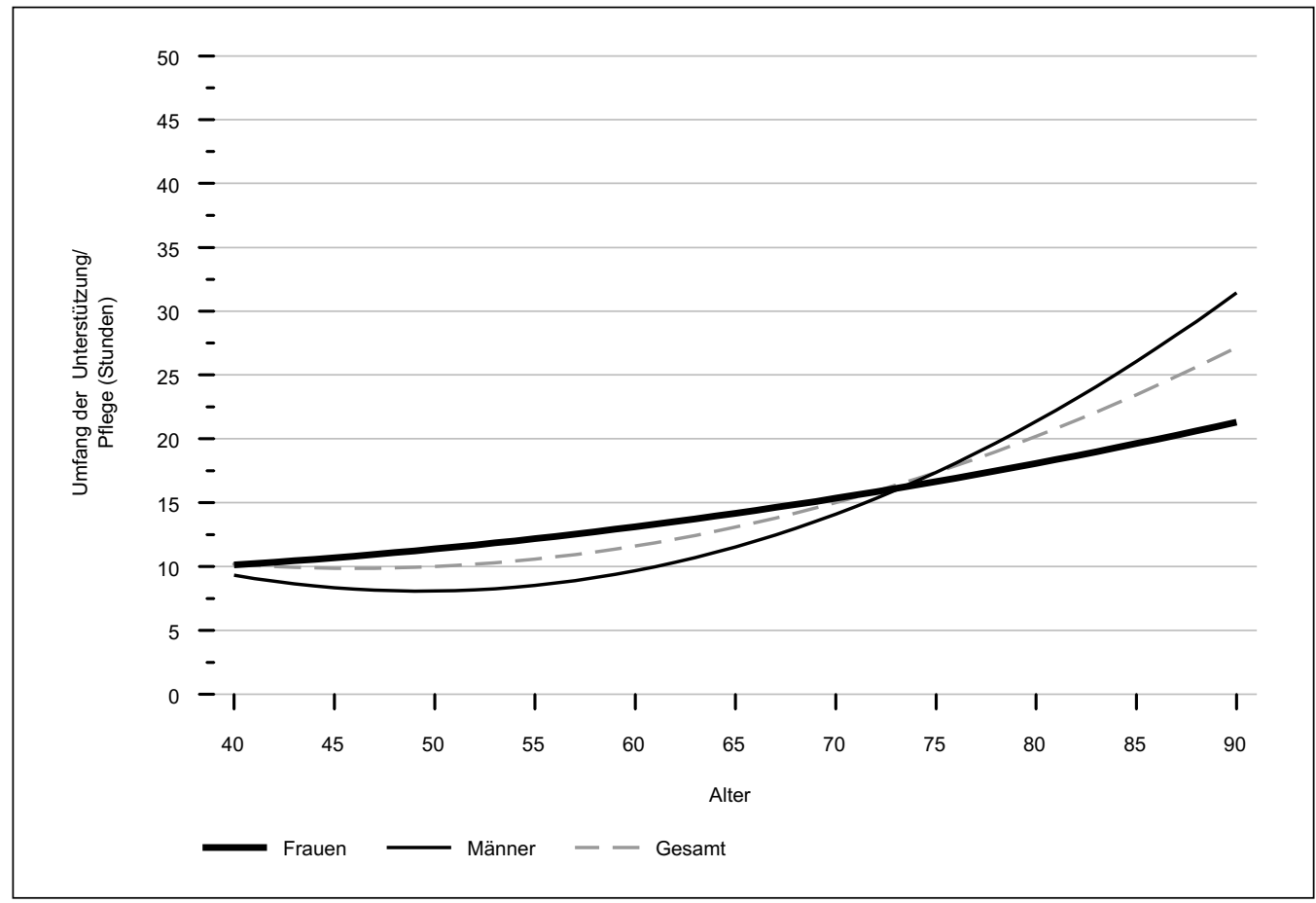

Quelle: DEAS 1996-2017, Schätzungen basieren auf $n=3991$ Studienteilnehmenden mit insgesamt $n=5360$ Beobachtungen, kontrolliert für Region.

Bei Frauen steigt der mittlere Umfang geleisteter Unterstützung und/oder Pflege von zehn Wochenstunden (im Alter von 40 Jahren) auf 13 Wochenstunden an (im Alter von 6o Jahren). Bei Männern steigt der Umfang von neun Wochenstunden (im Alter von 40 Jahren) auf zehn Wochenstunden an (im Alter von 6o Jahren). Im Alter von 6o Jahren unterscheiden sich die geschätzten Umfänge für Frauen und Männer signifikant. Die geschlechtsspezifischen Unterschiede im geschätzten Alternsverlauf sind signifikant. 
unterschiedes bis zum 56. Lebensjahr (Unterschied von vier Wochenstunden). Ab dem 66. Lebensjahr zeigt sich kein Unterschied mehr im zeitlichen Betreuungsaufwand von Frauen und Männern. Die in Abbildung 6-7 sichtbaren Unterschiede im hohen Alter sind - vermutlich aufgrund der vergleichs- weise geringen Befragtenzahlen - statistisch nicht signifikant. Ob sich dieses geschlechtsspezifische (Verlaufs-)Muster über die Geburtskohorten hinweg gewandelt hat, kann - ebenfalls aufgrund der geringen Fallzahlen - nicht untersucht werden.

\subsection{Diskussion}

\section{Zusammenfassung}

Die Ergebnisse belegen deutliche Geschlechterunterschiede in der Ausübung von Sorgetätigkeiten, die sich sowohl im Alter von 60 Jahren als auch im Verlauf der zweiten Lebenshälfte zeigen (erste Forschungsfrage). Darüber hinaus gibt es punktuelle Hinweise auf eine in den später geborenen Kohorten stattfindende Angleichung dieser Unterschiede zwischen Frauen und Männern (zweite Forschungsfrage).

Konkret hat sich gezeigt, dass Frauen nicht nur häufiger Enkelkinder betreuen und hilfsbedürftige Familienangehörige oder Personen aus der Nachbarschaft oder dem Freundes- und Bekanntenkreis unterstützen und pflegen als Männer. Frauen tun dies auch zeitintensiver, früher in der zweiten Lebenshälfte und tendenziell über eine längere Zeitspanne hinweg als Männer. Während bei Frauen diese Aufgaben bereits in der späten Erwerbsphase beginnen, konzentrieren sich diese Tätigkeiten bei den Männern eher auf die Zeit nach dem Renteneintritt. So kommt es mit Beginn der zweiten Lebenshälfte typischerweise zunächst $\mathrm{zu}$ einem Auseinanderdriften der geschlechtsspezifischen Verläufe, das etwa Mitte 50 (Unterstützung und Pflege) und Anfang 60 (Enkelbetreuung) seinen Höhepunkt erreicht. In dieser Altersphase fallen die Unterschiede in der Übernahme von Sorgetätigkeiten zwischen Frauen und Männern am größten aus. Danach setzt eine Angleichung der Unterschiede ein. Dieser Prozess geht allerdings weniger auf ein aktives „Aufholen“ der Männer zurück, sondern ist vielmehr das Resultat eines allgemei- nen altersbedingten Rückgangs, der Frauen stärker betrifft als Männer.

Hinweise für einen gesellschaftlichen Wandel dieser geschlechtsspezifischen Muster und $\mathrm{Al}$ ternsverläufe finden sich bislang nur für die Enkelbetreuung: Für die Kohorte der 1950 bis 1959 Geborenen konnte - im Vergleich zur Kohorte 1930 bis 1939 - eine Angleichung zwischen den Geschlechtern festgestellt werden. Diese Angleichung beruht auf zwei Verschiebungen: Erstens darauf, dass sich Männer späterer Geburtsjahrgänge stärker an der Enkelbetreuung beteiligen als früher geborene Männer und zweitens, dass Frauen der jüngeren Kohorten seltener betreuen. Daneben hat sich für beide Geschlechter gleichermaßen die Hauptphase der Enkelbetreuung in ein deutlich höheres Lebensalter verlagert: Der soziale Wandel zeigt sich in einer Verlagerung von durchschnittlich zehn Jahren zwischen der 1930er und der 1950er Kohorte. Diese biografische Verlagerung hat dazu geführt, dass ein Großteil der Enkelbetreuungen nunmehr im Rentenalter stattfindet.

Eine vergleichbare Annäherung der Geschlechterunterschiede ist für die Unterstützung und Pflege kranker verwandter oder nicht-verwandter Personen nicht zu beobachten: Zwar werden diese Arbeiten in der Kohorte der 1950 bis 1959 Geborenen häufiger übernommen als in der Kohorte der 1930 bis 1939 Geborenen und es ist zu einer stärkeren Konzentration auf eine bestimmte Altersphase - um das 60. Lebensalter herum - gekommen. Doch die Unterschiede zwischen den Geschlechtern bestehen nach wie vor. 


\section{Interpretation}

Anhand der Untersuchung alterstypischer Sorgetätigkeiten bestätigt sich auch für das höhere Lebensalter und insbesondere die späte Erwerbsphase die bekannte klassische geschlechtsspezifische Rollen- und Arbeitsverteilung: Familienbezogene Arbeiten liegen - wahrscheinlich in Fortführung der in vorangegangenen Lebens- und Familienphasen praktizierten Arbeitsteilung - auch in diesem Alter eher im Verantwortungsbereich der Frauen. Frauen pflegen familiale und andere enge Beziehungen stärker, indem sie häufiger, zeitintensiver und - wie die Längsschnittanalysen in diesem Band besonders eindrücklich zeigen - dauerhafter als gleichaltrige Männer Unterstützung, Betreuung bis hin zu krankheitsverursachter Pflege im privaten Umfeld leisten.

Dass sich bei Frauen eine Häufung der Betreuungs- und Unterstützungsleistungen in der späten Erwerbsphase zeigt, während sich Männer erst später (zumeist im frühen Rentenalter) verstärkt beteiligen, hat einerseits demografische Ursachen: Dass Frauen in einem jüngeren Alter Enkelbetreuung aufnehmen als Männer ist teilweise auf ihr geringeres Alter bei Geburt des ersten Kindes zurückzuführen sowie den typischen Altersabstand in Ehen und Partnerschaften von etwa drei Jahren. Männer sind bei Geburt ihres ersten Enkelkindes im Schnitt drei Jahre älter als Frauen (54 Jahre zu 51 Jahre, vgl. Mahne \& Klaus 2017: 236). Die generell seltenere Enkelbetreuungsübernahme unter Männern hat möglicherweise auch damit zu tun, dass Männer etwas häufiger kinderlos sind und damit auch häufiger als Frauen keine Enkelkinder haben. So haben elf Prozent der 70- bis 85-jährigen Frauen keine Enkelkinder im Vergleich zu 18 Prozent der Männer (Deutscher Alterssurvey 2014, eigene Auswertungen). Das grundsätzlich stärkere Engagement von Frauen in den beiden betrachteten Sorgebereichen ist zugleich Ausdruck geschlechtsspezifischer Muster der Aufteilung von bezahlter Erwerbs- und unbezahlter Sorgetätigkeit. Die über Jahrzehnte hinweg bestehende geringere Erwerbsbeteiligung der Frauen, die sich trotz stei- gender Tendenz auch nach wie vor in der späten Erwerbsphase zeigt, forciert geschlechtsspezifische Erwartungsmuster: Danach sind sie es, die sich stärker im Sorgebereich engagieren ebenso wie erwerbstätige Frauen eine bestehende Erwerbstätigkeit im Fall nachgefragter Betreuungs- oder Pflegeaufgaben eher reduzieren oder auch ganz aufgeben als Männer.

Auch die beschriebenen gesellschaftlichen Veränderungen im Verlauf der letzten Jahre sind zunächst Folge demografischer Verschiebungen: So geht der stattgefundene Aufschub der Enkelbetreuung in ein biografisch höheres Alter - zumindest partiell - direkt darauf zurück, dass heutige Eltern später Großeltern werden als noch vor einigen Jahren (Klaus \& Mahne 2017: 235). Das wiederum ist Folge der späteren Familiengründung. Allerdings kann die Abnahme der Geschlechterunterschiede in der Enkelkinderbetreuung auch als Ausdruck einer einsetzenden Aufweichung der geschlechtsspezifischen Aufgaben- und Rollenteilung interpretiert werden: Denn Frauen in den später geborenen Kohorten sind eben auch zu höheren Anteilen erwerbstätig als dies Frauen in den früher geborenen Kohorten waren. Parallel dazu engagieren sich Männer in den später geborenen Kohorten eher in der Enkelbetreuung, was bei ihren eigenen Vätern meist noch deutlich seltener der Fall war.

Sehr deutlich schlägt sich der demografische Wandel - insbesondere die zunehmende Hochaltrigkeit - in einem Wandel von Unterstützungsund Pflegetätigkeiten über die Generationen hinweg nieder: So ist die Wahrscheinlichkeit, die Tätigkeiten auszuüben, in den später geborenen Kohorten angestiegen ebenso wie es zu einer Verschiebung in ein höheres Alter gekommen ist. Da gerade unter den Ältesten der Anteil der Verwitweten zurückgegangen ist beziehungsweise sich die Verwitwung in ein höheres Alter verschoben hat (Engstler \& Klaus 2017: 205), ist davon auszugehen, dass die typischerweise im höheren Alter ausgeübte Pflege der Partnerin oder des Partners zugewonnen hat - indem sie stärker nachgefragt wird, aber auch aufgrund länger erhaltener funktionaler Gesundheit eher geleistet werden kann. 
Allerdings ist hier bei später geborenen Kohorten keine Angleichung zwischen Frauen und Männern festzustellen.

\section{Politische Implikationen}

Nicht zuletzt begründet durch die nach wie vor bestehenden und offenbar auch beständigen $\mathrm{Ge}$ schlechterunterschiede in der Übernahme verschiedener Sorgetätigkeiten, besteht weiterhin dringender politischer Handlungsbedarf, um einer einseitigen Be- oder gar Überlastung von Frauen entgegenzuwirken. Ein Ziel ist die Entlastung von Frauen aber auch von Männern, die im hohen (Erwerbs-)Alter Sorgetätigkeiten übernehmen (wollen) und möglicherweise zugleich erwerbstätig sind (sein wollen).

Um das zu erreichen, sind in erster Linie bereits bestehende Maßnahmen und Vorhaben weiter voranzutreiben, die auf den Ausbau institutioneller Betreuungseinrichtungen (in den Bereichen Kinder- und Altenversorgung) und die Ausweitung des Angebots professioneller Dienstleister zielen. Deren Verfügbarkeit kann in ergänzender Form dazu beitragen, dass der Umfang der informell übernommenen Sorgetätigkeiten besser auf das jeweils leistbare oder gewollte Maß zugeschnitten werden kann. Oder aber institutionelle oder professionelle Betreuungsmöglichkeiten springen gänzlich ein, wenn anstehende Sorgeaufgaben - aus welchen Gründen auch immer - (temporär) nicht von den 40 - bis 90 -Jährigen privat übernommen werden können.

Darüber hinaus sind, nicht nur unter der Prämisse der Entlastung der Frauen, sondern auch im Sinne einer Geschlechtergerechtigkeit, Männer stärker für relevante Sorgebereiche zu gewinnen. Auf diese Weise lassen sich nachgefragte Betreuungs- und Pflegeaufgaben im privaten Umfeld auf mehrere Schultern verteilen und so das Risiko der Überlastung einzelner Hauptverantwortlicher mindern. Eine verstärkte Einbindung von Personen aus der Nachbarschaft und aus dem Freundes- und Bekanntenkreis könnte ebenfalls zur Ent- lastung beitragen, allerdings muss auch hier der Aspekt der Geschlechtergerechtigkeit im Blick behalten werden. Ohne politische Gegensteuerung würde die bei Frauen höhere Wahrscheinlichkeit der Übernahme von Pflege und Betreuung im Freundeskreis und in der Nachbarschaft wiederum die traditionelle Arbeitsteilung zementieren und die Doppelbelastungen für Frauen dauerhaft im Verlauf der zweiten Lebenshälfte verschärfen. Grundlegend für mehr Gleichberechtigung im Bereich der gesellschaftlich höchst relevanten Sorgearbeit ist es, weiter an der Vermittlung und Etablierung modernisierter Rollenbilder zu arbeiten - bereits in frühen Lebens- und Familienphasen und nicht zuletzt unterstützt durch eine weitere Förderung der Frauenerwerbsbeteiligung.

Parallel dazu sind nach wie vor Bedingungen zu schaffen und zu verbessern, die es erwerbstätigen Frauen und Männern gleichermaßen ermöglichen, Sorgeaufgaben zu übernehmen. Neben der Gewährleistung von Arbeitsplatzsicherheit und finanzieller Absicherung in Phasen temporärer Erwerbsreduktion steht hier vor allem eine Flexibilisierung von Arbeitszeiten und -ort im Vordergrund. Denn ein Vereinbarkeitsproblem besteht selten allein aufgrund der benötigten Zeit für Sorgetätigkeiten, sondern auch aufgrund der Tatsache, dass Betreuungs- und Pflegeverpflichtungen zeitlich nicht gut planbar sind und oft kurzfristiges Handeln erfordern. Zudem darf neben den Ansprüchen der bezahlten Arbeit auch der Stress und die z.B. emotional teils hohe Belastung durch unbezahlte Arbeit nicht vergessen werden. Auch in diesem Zusammenhang sind Unterstützungs- und Entlastungmaßnahmen für stark beanspruchte Sorgeleistende zu konzipieren.

Politische Maßnahmen, die etwa eine Übernahme von Enkelkinderbetreuung durch die Großeltern unterstützen, sollten umgekehrt auch jeweils daraufhin geprüft werden, wie gegebenenfalls eine Erwerbstätigkeit der Großeltern dadurch beeinflusst ist. Aufgrund der insgesamt geringeren Rentenanwartschaften von Frauen wäre es aus unserer Sicht ein falsches Signal, Großmütter zu ermutigen, ihre Erwerbstätigkeit zugunsten der En- 
kelkinderbetreuung frühzeitig zu beenden oder zu reduzieren, mit den entsprechenden nachteiligen Konsequenzen für die individuellen Alterseinkommen. Auch der derzeit oft beklagte Arbeitskräftemangel, bei gleichzeitigem Trend zum längeren Arbeiten für bestimmte Gruppen, sollte nicht durch Anreize zur Beendigung der beruflichen Tätigkeit aufgrund der Übernahme familienbezogener Verpflichtungen verschärft werden. Vor dem Hintergrund der Tatsache, dass viele Großeltern die Enkelkinderbetreuung übernehmen, um ihre erwachsenen Kinder zu entlasten und ihnen für die berufliche Karriere „den Rücken freizuhalten“, geht es auch immer darum, durch staatliche Infrastruktur Betreuungsarrangements zu schaffen, die allen Generationen die Arbeitsmarktpartizipation ermöglichen. Vergleichbar zu den Maßnahmen, Väter stärker zur Übernahme von Erziehungsaufgaben zu motivieren, ist auch hier zu überlegen, die Enkelkinderbetreuung auf mehr Personen zu verteilen. Zentral ist dabei, dass Maßnahmen zur Vereinbarkeit von Großelternschaft und Berufstätigkeit insbesondere auch Großväter erreichen, die sich dann biografisch früher an der Enkelkin- derbetreuung beteiligen. Dies wäre insbesondere vor dem Hintergrund sich langsam wandelnder Geschlechternormen und modernisierter Rollenbilder erfolgversprechend.

Insgesamt ist es aus unserer Sicht von großer Bedeutung, dass politische Maßnahmen zur Verbesserung der Vereinbarkeit von unbezahlten familialen Sorgetätigkeiten und bezahlten Erwerbstätigkeiten nicht verkürzt auf jene Personen zugeschnitten werden, die derzeit mehrheitlich vor der Frage der Vereinbarkeit stehen. Das heißt, entsprechende Maßnahmen und Unterstützungsangebote sind grundsätzlich an alle Personen zu adressieren und nicht nur an Frauen. Von verbesserten Rahmenbedingungen sollten erwerbstätige Männer, die Angehörige betreuen und pflegen, ebenso profitieren wie erwerbstätige Frauen. Ohne politische Steuerung ist eine Gleichstellung der Geschlechter in Bezug auf familienbezogene Aufgaben und Sorgetätigkeiten in Deutschland jedenfalls kaum zu erreichen. Und bislang gestaltet sich in diesem Bereich die Situation späterer Jahrgänge kaum besser als die der früheren Jahrgänge von Frauen und Männern.

\section{Literatur}

Bestmann, B., Wüstholz, E., \& Verheyen, F. (2014). Pflegen: Belastung und sozialer Zusammenhalt. Eine Befragung zur Situation von pflegenden Angehörigen [WINEG Wissen 4]. Hamburg: Techniker Krankenkasse.

Bundesministerium für Familie, Senioren, Frauen und Jugend (2018). Kindertagesbetreuung Kompakt. Ausbaustand und Bedarf 2017. Berlin: Bundesministerium für Familie, Senioren, Frauen und Jugend.

Bolin, K., Lindgren, B., \& Lundborg, P. (2008). Informal and formal care among single living elderly in Europe. Health economics, 17(3), 393-409.

Brandt, M., Haberkern, K., \& Szydlik, M. (2009). Intergenerational help and care in Europe. European Sociological Review, 25(5), 585-601.

Brettschneider, A., \& Klammer, U. (2016). Lebenswege in die Altersarmut. Biografische Analysen und sozialpolitische Perspektiven. Berlin: Duncker \& Humblot.

Brüderl, J. (2010). Kausalanalyse mit Paneldaten. In: C. Wolf \& H. Best (Hrsg.) Handbuch der sozialwissenschaftlichen Datenanalyse (S. 963-994). Wiesbaden: VS Verlag für Sozialwissenschaften.

Bundesministerium für Familie, Senioren, Frauen und Jugend (2018). Kindertagesbetreuung Kompakt. Ausbaustand und Bedarf 2017. Berlin: Bundesministerium für Familie, Senioren, Frauen und Jugend. 
Deindl, C., \& Brandt, M. (2011). Financial support and practical help between older parents and their middle-aged children in Europe. Ageing \& Society, 31(04), 645-662.

Deutscher Bundestag (2017). Zweiter Gleichstellungsbericht der Bundesregierung. Erwerbs- und Sorgearbeit gemeinsam neu gestalten. (Drucksache 18/12840). Berlin: Deutscher Bundestag.

Engstler, H., \& Klaus, D. (2017). Auslaufmodell ,traditionelle Ehe'? Wandel der Lebensformen und der Arbeitsteilung von Paaren in der zweiten Lebenshälfte. In: K. Mahne, J. K. Wolff, J. Simonson \& C. TeschRömer (Hrsg.) Altern im Wandel. Zwei Jahrzehnte Deutscher Alterssurvey (DEAS) (S. 201-213). Wiesbaden: Springer VS.

Engstler, H., \& Tesch-Römer, C. (2017). Zeitverwendung von Erwachsenen, die ein Haushaltsmitglied pflegen. In: Statistisches Bundesamt (Hrsg.) Wie die Zeit vergeht: Analysen zur Zeitverwendung in Deutschland. Beiträge zur Ergebniskonferenz der Zeitverwendungserhebung 2012/2013 am 5./6. Oktober 2016 in Wiesbaden (S. 229-244). Wiesbaden: Statistisches Bundesamt.

Frerk, T., \& Leitner, S. (2017). Zur Vereinbarkeit von Pflege und Beruf: Quantitative und qualitative Perspektiven. Sozialer Fortschritt, 66(3-4), 267-283. doi: 10.3790/sfo.66.3-4.267.

Geyer, J. (2016). Informell Pflegende in der deutschen Erwerbsbevölkerung: Soziodemografie, Pflegesituation und Erwerbsverhalten. In: Zentrum für Qualität in der Pflege (Hrsg.) ZQP-Themenreport. Vereinbarkeit von Beruf und Pflege (S. 24-43). Berlin: Zentrum für Qualität in der Pflege.

Geyer, J., \& Schulz, E. (2014). Who cares ? Die Bedeutung der informellen Pflege durch Erwerbstätige in Deutschland. DIW Wochenbericht, 81(14), 294-301.

Hank, K., \& Buber, I. (2009). Grandparents caring for their grandchildren. Findings From the 2004 Survey of Health, Ageing, and Retirement in Europe. Journal of Family Issues, 30(1), 53-73.

Henz, U. (2004). The effects of informal care on paid-work participation in Great Britain: a lifecourse perspective. Ageing \& Society, 24(06), 851-880.

Hobler, D., Klenner, C., Pfahl, S., Sopp, P., \& Wagner, A. (2017). Wer leistet unbezahlte Arbeit ? Hausarbeit, Kindererziehung und Pflege im Geschlechtervergleich. Aktuelle Auswertungen aus dem WSI GenderDatenPortal [WSI Report Nr. 35]. Düsseldorf: Wirtschafts- und Sozialwissenschaftliches Institut (WSI) in der Hans-Böckler-Stiftung.

Igel, C., \& Szydlik, M. (2011). Grandchild care and welfare state arrangements in Europe. Journal of European Social Policy, 21(3), 210-224. doi: 10.1177/0958928711401766.

Kalmijn, M., \& Saraceno, C. (2008). A comparative perspective on intergenerational support. European Societies, $10(3), 479-508$.

Keck, W. (2016). Was kommt nach der Pflege? Die Pflege eines Angehörigen senkt Beschäftigungschancen von Pflegepersonen nachhaltig. Sozialer Fortschritt, 65(5), 112-119.

Keck, W. (2012). Die Vereinbarkeit von häuslicher Pflege und Beruf. Bern: Huber. doi: 10.3790/sfo.65.5.112.

Klaus, D. (2009). Why Do Adult Children Support Their Parents? Journal of Comparative Family Studies, $4 O(2), 227-241$.

Klaus, D., \& Tesch-Römer, C. (2017). Pflege und Unterstützung bei gesundheitlichen Einschränkungen: Welchen Beitrag leisten Personen in der zweiten Lebenshälfte für andere ? In: K. Mahne, J. K. Wolff, J. Simonson \& C. Tesch-Römer (Hrsg.) Altern im Wandel. Zwei Jahrzehnte Deutscher Alterssurvey (DEAS) (S. 185-200). Wiesbaden: Springer VS.

Leopold, T., \& Raab, M. (2011). Kurzzeitreziprozität in späten Eltern-Kind-Beziehungen. In: P. A. Berg (Hrsg.) Reproduktion von Ungleichheit durch Arbeit und Familie (S. 93-119). Wiesbaden: VS Verlag für Sozialwissenschaften.

Leopold, T., Raab, M., \& Engelhardt, H. (2014). The transition to parent care: Costs, commitments, and caregiver selection among children. Journal of Marriage and Family, 76(2), 300-318. 
Leopold, T., \& Skopek, J. (2015). The Delay of Grandparenthood: A Cohort Comparison in East and West Germany. Journal of Marriage and Family, 77(2), 441-460.

Mahne, K., \& Klaus, D. (2017). Zwischen Enkelglück und (Groß-)Elternpflicht - die Bedeutung und Ausgestaltung von Beziehungen zwischen Großeltern und Enkelkindern. In: K. Mahne, J. K. Wolff, J. Simonson \& C. Tesch-Römer (Hrsg.) Altern im Wandel. Zwei Jahrzehnte Deutscher Alterssurvey (DEAS) (S. 231-245). Wiesbaden: Springer VS.

Nowossadeck, S., Engstler, H., \& Klaus, D. (2016). Pflege und Unterstützung durch Angehörige. [Report Altersdaten 1/2016]. Berlin: Deutsches Zentrum für Altersfragen.

Rothgang, H., Iwansky, S., Müller, R., Sauer, S., \& Unger, R. (2010). Barmer GEK Pflegereport 2010. Schwerpunktthema: Demenz und Pflege [Schriftenreihe zur Gesundheitsanalyse Band 5]. Schwäbisch Gmünd: BARMER GEK.

Runde, P., Giese, R., Kaphengst, C., \& Hess, J. (2009). AOK-Trendbericht Pflege II. Entwicklung in der häuslichen Pflege seit Einführung der Pflegeversicherung. Hamburg: Universität Hamburg, Fakultät Wirtschafts- und Sozialwissenschaften.

Schäufele, M., Köhler, L., \& Hendlmeier, I. (2016). Erwerbstätigkeit und Pflege von Menschen mit Demenz: Ergebnisse einer bundesweiten Repräsentativstudie. In: Zentrum für Qualität in der Pflege (Hrsg.) Vereinbarkeit von Beruf und Pflege (S. 44-59). Berlin: Zentrum für Qualität in der Pflege.

Schröder, A. (2007). Prinzipien der Panelanalyse. In: S. Albers, D. Klapper, U. Konradt, A. Walter \& J. Wolf (Hrsg.) Methodik der empirischen Forschung (S. 261-276). Wiesbaden: Springer Gabler.

Schwarz, N., \& Schwahn, F. (2016). Entwicklung der unbezahlten Arbeit privater Haushalte. Bewertung und Vergleich mit gesamtwirtschaftlichen Größen. WISTA - Wirtschaft und Statistik, 2016(2), 35-51.

Schwinger, A., Tsiasioti, C., \& Klauber, J. (2016). Unterstützungsbedarf in der informellen Pflege - eine Befragung pflegender Angehöriger. In: K. Jacobs, A. Kuhlmey, S. Greß, J. Klauber \& A. Schwinger (Hrsg.) Pflege-Report 2016 (S. 189-216). Stuttgart: Schattauer.

Spiess, C. K., \& Schneider, A. U. (2003). Interactions between care-giving and paid work hours among European midlife women, 1994 to 1996. Ageing \& Society, 23(1), 41-68.

Statistisches Bundesamt (2018). Statistiken der Kinder- und Jugendhilfe. Kinder und tätige Personen in Tageseinrichtungen und in öffentlich geförderter Kindertagespflege am 01. 03. 2017. Wiesbaden: Statistisches Bundesamt.

Statistisches Bundesamt (2017). Pflegestatistik 2015. Wiesbaden: Statistisches Bundesamt.

Stuifbergen, M. C., Delden, J. J. M. van, \& Dykstra, P. A. (2008). The implications of today's family structures for support giving to older parents. Ageing \& Society, 28(3), 413-434.

TNS Infratest Sozialforschung (2011). Abschlussbericht zur Studie „Wirkungen des Pflege-Weiterentwicklungsgesetzes". Berlin: Bundesministerium für Gesundheit.

Vogel, C., \& Künemund, H. (2018). Armut im Alter. In: P. Böhnke, J. Dittmann \& J. Goebel (Hrsg.) Handbuch Armut (S. 144-153). Leverkusen: Barbara Budrich.

Vogel, C., \& Scherger, S. (2018). Einleitung. In: C. Vogel \& S. Scherger (Hrsg.) Arbeit im Alter. Zur Bedeutung bezahlter und unbezahlter Tätigkeiten in der Lebensphase Ruhestand (S.1-25). Wiesbaden: Springer VS. 
Open Access Dieses Kapitel wird unter der Creative Commons Namensnennung 4.0 International Lizenz (http:// creativecommons.org/licenses/by/4.0/deed.de) veröffentlicht, welche die Nutzung, Vervielfältigung, Bearbeitung, Verbreitung und Wiedergabe in jeglichem Medium und Format erlaubt, sofern Sie den/die ursprünglichen Autor(en) und die Quelle ordnungsgemäß nennen, einen Link zur Creative Commons Lizenz beifügen und angeben, ob Änderungen vorgenommen wurden.

Die in diesem Kapitel enthaltenen Bilder und sonstiges Drittmaterial unterliegen ebenfalls der genannten Creative Commons Lizenz, sofern sich aus der Abbildungslegende nichts anderes ergibt. Sofern das betreffende Material nicht unter der genannten Creative Commons Lizenz steht und die betreffende Handlung nicht nach gesetzlichen Vorschriften erlaubt ist, ist für die oben aufgeführten Weiterverwendungen des Materials die Einwilligung des jeweiligen Rechteinhabers einzuholen. 